\title{
End-to-End Assembly of Shape-Controlled Nanocrystals via a Nanowelding Approach Mediated by Gold Domains
}

\author{
By Albert Figuerola, Isabella R. Franchini, Angela Fiore, Rosanna Mastria, \\ Andrea Falqui, Giovanni Bertoni, Sara Bals, Gustaaf Van Tendeloo, \\ Stefan Kudera, Roberto Cingolani, and Liberato Manna*
}

The assembly of nanostructures into controlled 2D and 3D superstructures over large length scales is one of the main challenges in chemistry and materials science, as this is of great importance for the construction of materials with tailored/ improved functionality. This can only be realized if one can finely govern the currently known mechanisms for nanometer-scale assembly, ${ }^{[1]}$ and if new assembly concepts are introduced, which can lead to previously unforeseen models of organization.

So far several types of assemblies have been realized with shape-controlled nanocrystals following many approaches. Colloidal nanorods for instance have been aligned in both vertically ${ }^{[2]}$ and laterally ${ }^{[3]}$ ordered arrays using a wide variety of techniques, which exploited Van der Waals interactions among nanorods, capillary forces, interaction with applied fields, and substrate templating effects. End-to-end assembly of nanorods, however, remains a challenge. ${ }^{[4]}$ So far, it has been achieved mainly by exploiting the higher reactivity of the nanorod end tips, which allows them to be selectively functionalized with molecules that mediate end-to-end nanorod organization. ${ }^{[5]}$ Recently, selective growth of Au tips on semiconductor nanorods has also been proposed as a way of improving end-to-end assembly, as functionalization of the $\mathrm{Au}$ domains at the nanorod tips with molecules capable of mutual recognition could lead to chainlike structures. ${ }^{[6]}$

[*] Dr. A. Figuerola, I. R. Franchini, A. Fiore, Dr. S. Kudera,

Prof. R. Cingolani, Dr. L. Manna

National Nanotechnology Laboratory of CNR-INFM,

Unità di Ricerca IIT

Distretto Tecnologico ISUFI, via per Arnesano

km 5, I-73100 Lecce (Italy)

Fax: $(+39) 0832298237$

E-mail: liberato.manna@unile.it

Dr. A. Figuerola, A. Fiore, R. Mastria, Prof. R. Cingolani

Scuola Superiore ISUFI; University of Salento

Distretto Tecnologico ISUFI, via per

Arnesano km 5, I-73100 Lecce (Italy)

Dr. A. Falqui, Dr. G. Bertoni

Italian Institute of Technology (IIT)

Via Morego 30, I-16163 Genova (Italy)

Prof. S. Bals, Prof. G. Van Tendeloo

EMAT, University of Antwerp

Groenenborgerlaan 171, B-2020 Antwerp (Belgium)

DOI: 10.1002/adma.200801928
Less studied is the assembly of branched nanostructures such as tetrapod-shaped nanocrystals, ${ }^{[7]}$ mainly because it is harder to realize superstructures with such nanocrystals. Nevertheless, some studies have been published concerning the controlled deposition of tetrapods on substrates. ${ }^{[8,9]}$ It would be desirable on the other hand to realize complex 3D networks of branched nanocrystals joined to each other via their tips, as this might lead to materials with controlled porosity, mechanical and electronic properties; such materials might even be used as scaffolds.

Here, we report a general and simple approach in a liquid solution for the assembly of several shape-controlled semiconductor nanocrystals, such as bullets, rods, and tetrapods. We exploit the shape anisotropy of nanocrystals to grow small metallic Au nanoparticles on selected locations of their surface. We then use small amounts of molecular iodine $\left(I_{2}\right)$, which destabilize the Au tips and induce the coalescence of Au domains belonging to different nanocrystals, thus forming larger $\mathrm{Au}$ particles, each of them bridging two or more nanocrystals through their tips. Such a 'nanowelding' process leads to linear and cross-linked chainlike assemblies of nanorods, to propellerand flowerlike structures based on bullet-shaped nanocrystals, and to networks of tetrapods (see Scheme 1 and Fig. 1-3). The approach is selective towards the Au domains and takes place

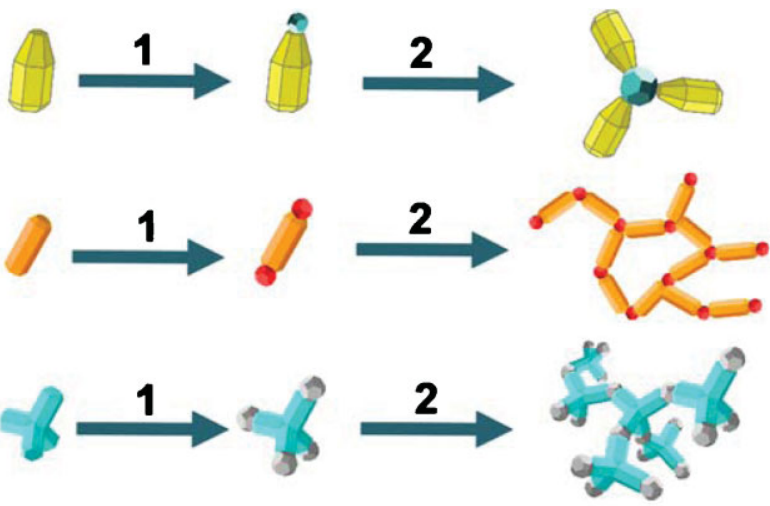

1.Precursors for gold nanocrystals: $\mathrm{AuCl}_{3}, \mathrm{TOAB}, \mathrm{DDA}$ 2.Iodine solution $: 0.1 \% \mathrm{l} / \mathrm{w} / \mathrm{v})$ molar ratio $\mathrm{NCs}: \mathrm{I}_{2}=1: 15$

Scheme 1. Assembly of bullet-, rod-, and tetrapod-shaped nanocrystals mediated by the coalescence of Au domains grown at their tips and then destabilized with the help of molecular iodine. The reactants for the gold growth are gold chloride $\left(\mathrm{AuCl}_{3}\right)$, tetraoctylammonium bromide (TOAB), and dodecylamine (DDA). 

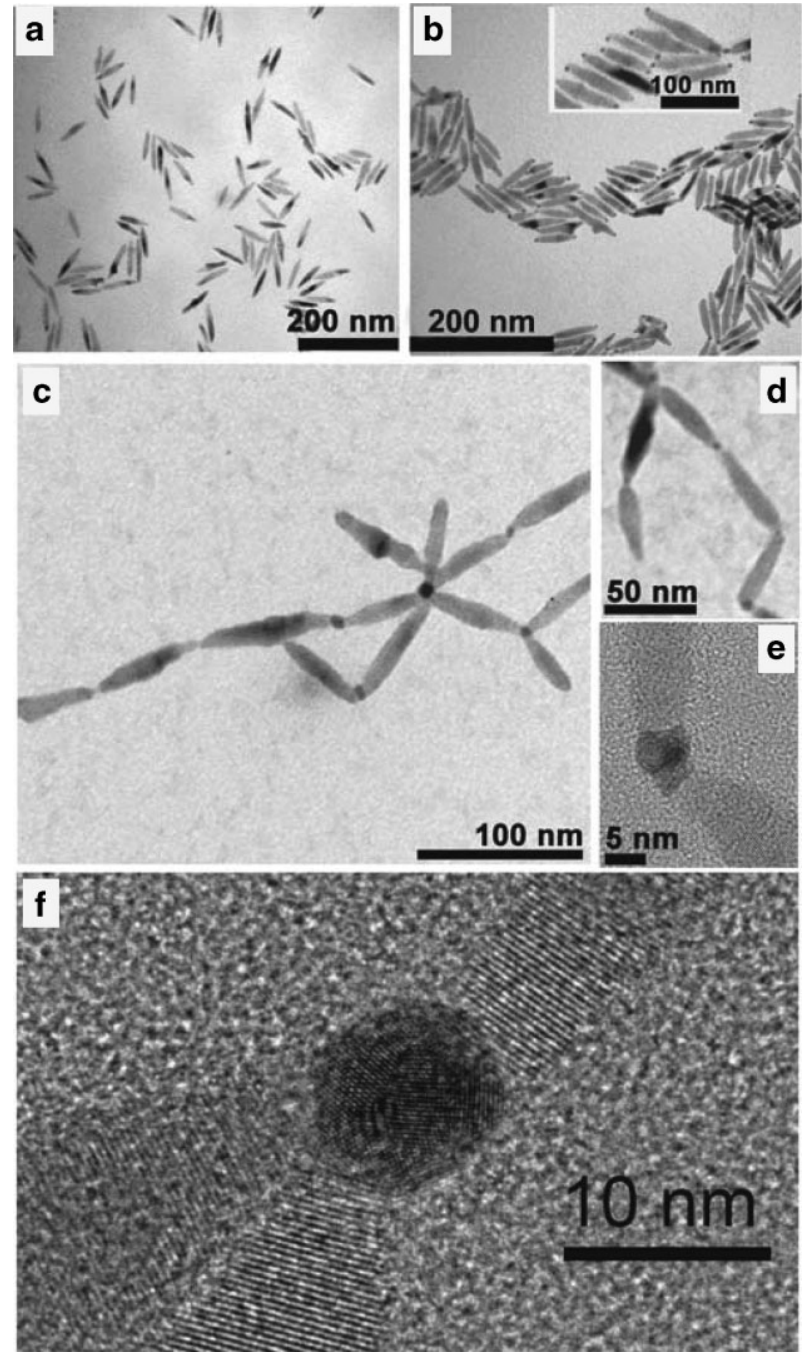

Figure 1. TEM images of a) CdSe nanorods, b) isolated Au-tipped CdSe nanorods, c) and d) iodine-induced chainlike aggregates of nanorods. e) HRTEM image of a $\mathrm{Au}$ junction area between two $\mathrm{CdSe}$ nanorods. f) HRTEM image of a $\mathrm{Au}$ junction area between three $\mathrm{CdSe}$ nanorods. Especially in the latter image it is possible to distinguish a polycrystalline structure for the Au nanoparticle. The starting semiconductor nanocrystals were prepared by a seeded-growth approach, which usually leads to nanocrystal samples with a relatively uniform distribution of shapes. The CdSe nanorods used here consist of spherical CdSe 'wurtzite' seeds onto which a thick rod-shaped CdSe shell was grown.

at room temperature in a solvent, in which as-grown nanocrystals are soluble (i.e. toluene or chloroform). Furthermore, such assemblies are stable in solution for a long time and withstand repeated precipitation and dissolution cycles, during which the $\mathrm{Au}$ welding among nanocrystals remains in place. The present strategy can be seen as an alternative method to the assembly mediated by organic molecules/biomolecules. ${ }^{[\mathrm{b}]}$ It introduces an inorganic and robust junction between nanocrystals, and hence avoids the use of molecular organic spacers for the assembly. Procedures involving the latter are indeed known to be tedious and difficult to upscale, and often involve reactions in an aqueous medium. While they offer some flexibility in the interparticle
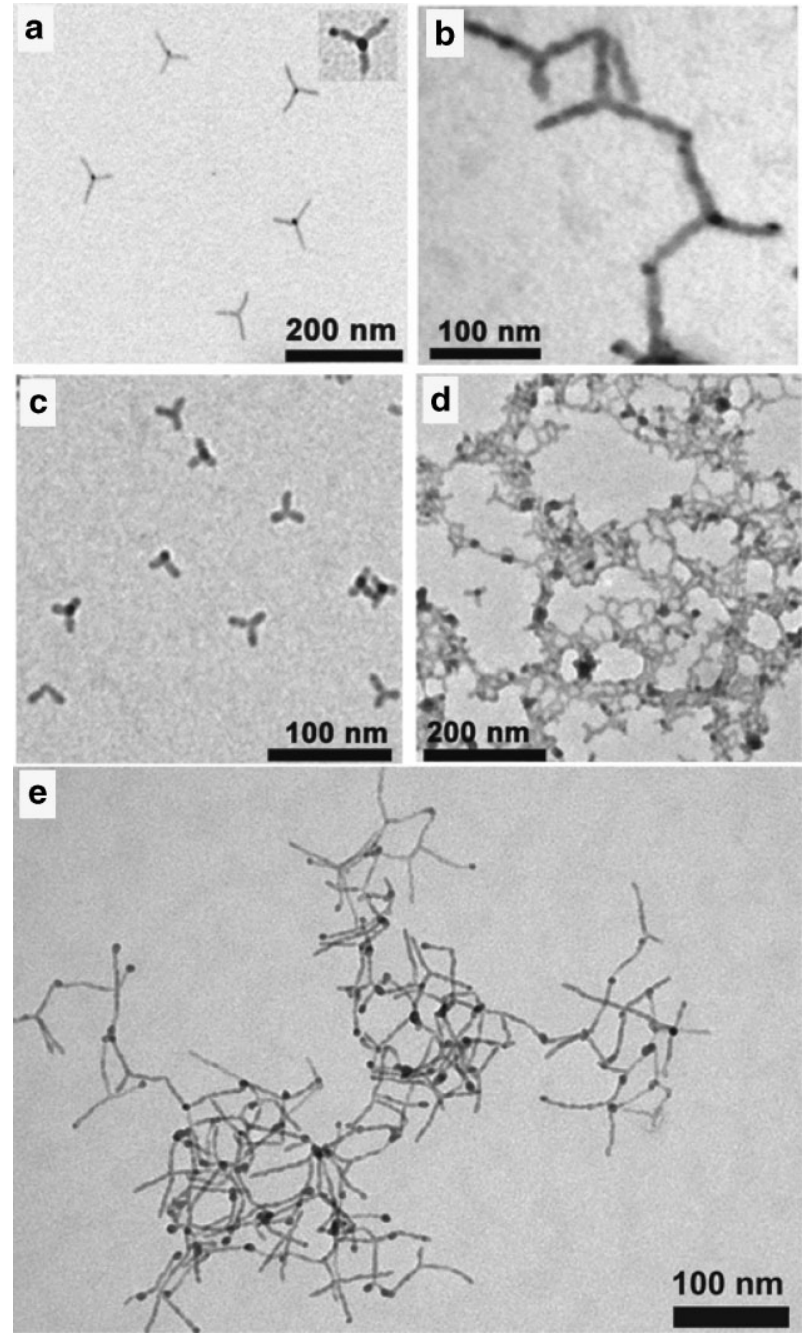

Figure 2. a) TEM images of $\mathrm{CdTe} @ \mathrm{CdS}$ tetrapods (i.e., central $\mathrm{CdTe}$ core and CdS arms); the inset shows an individual Au-tipped CdTe@CdS tetrapod. b) TEM image of detailed areas of CdTe@CdS tetrapod networks. The gold junctions between the arms of different tetrapods are clearly shown. c) TEM images of ZnTe@CdTe tetrapods. d) A wide field view of iodine-induced ZnTe@CdTe tetrapod networks. e) A wide field view of an isolated network of CdTe tetrapods, each of them carrying long arms. For the tetrapod networks all the starting nanocrystals were prepared by a seeded-growth approach. The CdTe@CdS and ZnTe@CdTe tetrapods consist of spherical $\mathrm{CdTe}$ and $\mathrm{ZnTe}$ 'sphalerite' seeds onto which four $\mathrm{CdS}$ or CdTe arms were grown, respectively. In the tetrapod nanocrystals in (e), the central core of each tetrapod is also composed of CdTe.

distance, the welding process presented here produces compact assemblies where the inter-particle distance is controlled only by the size of the $\mathrm{Au}$ junction.

A number of studies have been reported so far on the high reactivity of $\mathrm{Au}$ bulk surfaces and nanoparticles towards both molecular iodine $\left(\mathrm{I}_{2}\right)$ and iodide ions $\left(\mathrm{I}^{-}\right) \cdot{ }^{[10-15]}$ When $\mathrm{Au}$ nan oparticles are involved, chemisorbed iodine atoms are able to displace most or all ligands present on the nanoparticle surface, lower their surface charge, and therefore promote aggregation and coalescence that leads to the formation of larger particles, ${ }^{[10-12]}$ (see also Fig. S1 in the Supporting Information, 


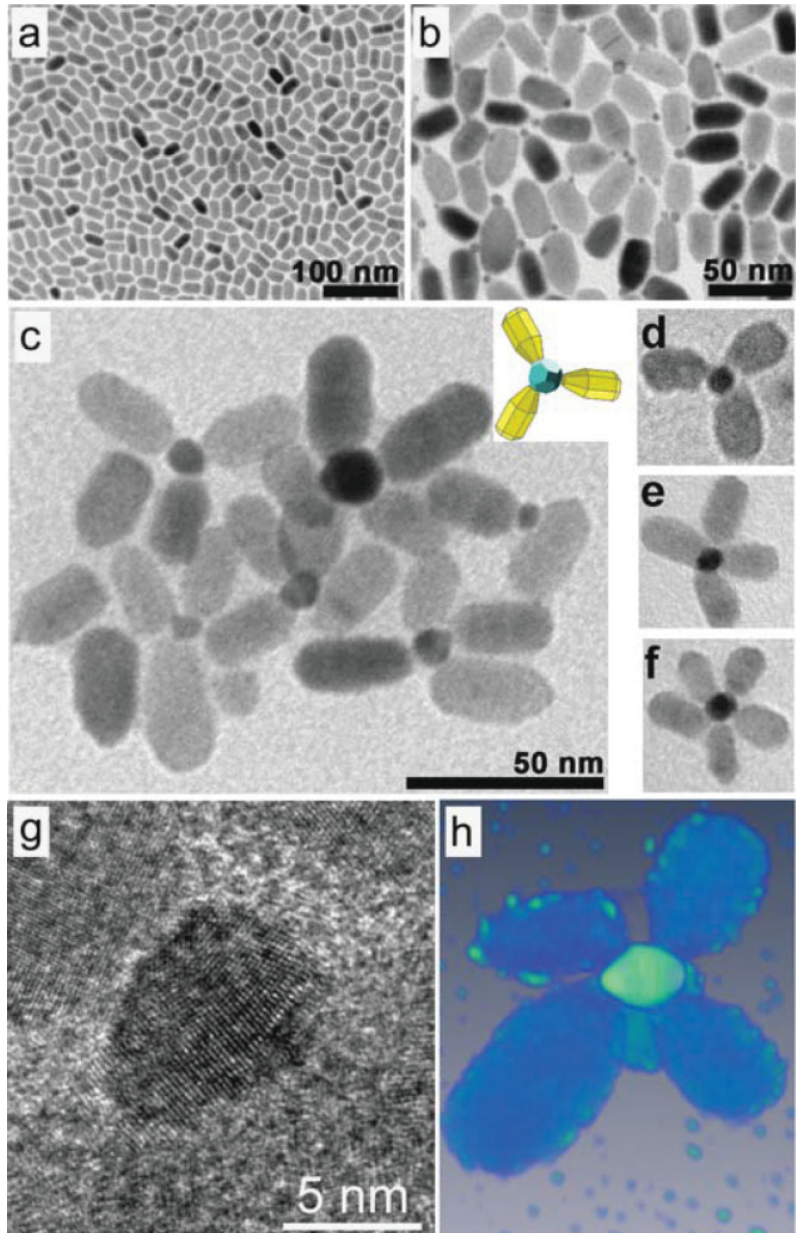

Figure 3. TEM images of a) bullet-shaped CdSe@CdS nanocrystals, b) Autipped bullet-shaped nanocrystals, c) iodine-induced propeller and flowerlike assemblies. d), e), and f) Isolated propeller- or flowerlike structures with 3, 4, and 5 petals, respectively. g) HRTEM image of the central part of a flowerlike structure where it is possible to see the multi-domain polycrystalline structure of the Au nanoparticle. h) A 3D reconstruction of a flowerlike structure based on electron tomography. The five CdSe@CdS petals form a $3 \mathrm{D}$ arrangement here (the entire tomography video is part of the Supporting Information). The bullet-shaped nanocrystals of panel (a) were prepared by a seeded-growth approach and consist of spherical CdSe 'wurtzite' seeds onto which a thick anisotropic CdS shell was grown.

SI). The chemistry of iodine/iodide has been exploited to induce morphological transformations, aggregation or dissolution of gold thin films and nanoparticles, ${ }^{[1-14]}$ and in the hydrometallurgical industry to recover gold from various materials (a greener alternative to cyanide) as stable $\mathrm{Au}$ coordination complexes. ${ }^{[16]}$ This inspired us in the search for a simple route for assembling nanocrystals via Au domains grown on selected locations on their surface.

The synthesis procedures of all starting nanocrystals used here were similar to those previously published by our group for the seeded growth of nanorods and tetrapods. ${ }^{[3 c]}$ These nanocrystals, as well as assemblies derived from them, were characterized by transmission electron microscopy (TEM) (see Fig. 1a, 2a,c and 3a for the initial nanocrystals). For the nucleation of gold domains on their tips, we followed the approach described by Banin and coworkers with slight modifications. ${ }^{[6]}$ Typical TEM images of the resulting Au-tipped nanocrystals are shown in Figure 1b, 2a (inset), and $3 \mathrm{~b}$.

In the case of Au-tipped nanorods, their reaction with $\mathrm{I}_{2}$ led to the formation of nanorod chains that were several tens of nanocrystals long, in which branching points were introduced whenever a Au domain was able to link more than two nanorod tips (Fig. 1c, 1d, and S2 in the SI). A high-resolution TEM (HRTEM) image of a $\mathrm{Au}$ nanoparticle bridging two CdSe nanorods is shown in Figure 1e, while Figure 1f is a HRTEM image of a $\mathrm{Au}$ junction between three CdSe nanorods. In particular, from Figure if one can see that the welding process among several Au domains that belong to different nanorods leads to a bigger, polycrystalline Au nanoparticle. On tetrapods, the welding of $\mathrm{Au}$ domains induced the organization of tetrapods into complex networks that could be extended up to several square micrometers (Fig. 2b,d,e, and S3 in the SI). It is likely that in solution such assemblies have a 3D globular structure. Their dimensions could be tuned by the amount of iodine added to the nanocrystal solution. Moreover, they often appeared to be stable in toluene, most likely because they could engulf large amounts of solvent in their interior. Only when a large excess of iodine was added to the solution, the size of the networks and the number of nanocrystals entrapped in them increased to an extent that a precipitate was formed during the reaction.

Additionally, the welding process can be controlled by choosing the number and position of the gold domains on the initial nanocrystals. Welding of bullet-shaped nanocrystals, each carrying a single Au nanoparticle most often localized at the bullet tip, led mainly to flower- and propellerlike structures (Fig. 3c). Although such structures tended to stay close to each other on the carbon support film of the grid when the solvent was evaporated, we could identify several of these isolated assemblies (see for instance Figure 3d, 3e, and 3f, and also Figure S4 of the SI for the assemblies obtained with other CdSe@CdS core@shell nanocrystals).

A HRTEM image of the central gold nanoparticle of a flowerlike structure is shown in Figure 3g. The Au region does not grow epitaxially on the CdS surface, since different relative crystallographic orientations at the junction between the semiconductor and the gold region have been observed in the sample, similar to what was reported by Banin and coworkers. ${ }^{\text {[6a] }}$ Furthermore, the vast majority of $\mathrm{Au}$ nanoparticles in such flowerlike assemblies display a polycrystalline structure (this is indeed the case of the particle imaged in Fig. 3g). The flowerlike assemblies were also studied by scanning electron microscopy (SEM). High-resolution SEM images (see Fig. S5 in the SI), as well as electron tomography reconstructions based on high angular annular dark-field scanning transmission electron microscopy (STEM) sequences of the flowerlike assemblies, prove that these structures are not limited to planar arrangements. Figure $3 \mathrm{~h}$ and the entire tomography video presented as Supporting Information show the three-dimensional layout of an isolated flowerlike assembly.

The room-temperature UV-vis absorption spectra of the bulletshaped core@shell nanocrystals, of the corresponding Au-tipped nanocrystals, and of the flowerlike assemblies in toluene solution are presented in Figure 4 (all samples had been previously cleaned 


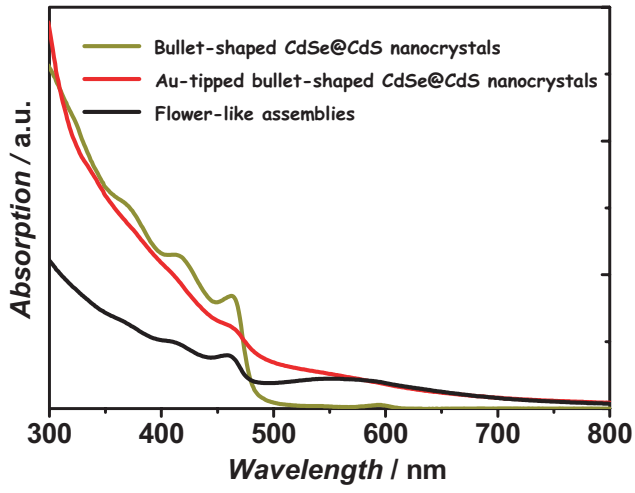

Figure 4. Room-temperature UV-visible absorption spectra of solutions containing bullet-shaped CdSe@CdS nanocrystals, Au-tipped bulletshaped nanocrystals, and the corresponding iodine-mediated flowerlike assemblies in toluene (the corresponding TEM images of these samples are shown in Fig. 3).

by precipitation with methanol and re-dissolution in toluene). The starting bullet-shaped CdSe@CdS core@shell nanocrystals show typical absorption peaks for this type of system. ${ }^{[3 c]}$ In the absorption spectrum of the solution containing the Au-tipped nanocrystals a wide plasmon band at around $560 \mathrm{~nm}$ confirms the presence of $\mathrm{Au}$ nanoparticles, albeit with a broad distribution of domain sizes. Also, the strong fluorescence of the starting CdSe@CdS nanocrystals is completely quenched in the corresponding Au-tipped sample, due to the direct contact of the semiconductor with the metallic nanoparticle (see Fig. S6 in the SI). ${ }^{[6]}$ After the reaction of the Autipped nanocrystals with iodine, a slight recovery of the original nanocrystal emission was usually observed, along with the appearance of a few isolated bullet-shaped nanocrystals without $\mathrm{Au}$ domains attached to them under TEM (see Fig. 3c). This indicated that some of the Au nanoparticles had been dissolved by iodine, thus restoring part of the fluorescence from the nanocrystals to which they were bound. The dissolution of probably the smallest $\mathrm{Au}$ domains present, together with the coalescence of most of the remaining Au domains into larger Au particles was also supported by the appearance of a well-defined Au plasmon band in the absorption spectrum, now peaked at a slightly longer wavelength (see Fig. 4).

In all the cases reported here, we are confident that coalescence of the gold particles at the nanocrystal tips is the cause of nanocrystal assembly. "Coagulation" of colloidal particles is a known phenomenon that has been studied both from a theoretical and experimental point of view. ${ }^{[17]}$ In our cases, the coagulation among Au nanoparticles seems to be confirmed by the polycrystalline structure of the Au particles that bind two or more nanocrystals in the assemblies reported here. Examples are the HRTEM images of Fig. 1e,f and 3g. Moreover, the final volume of the gold particles acting as junctions was verified to be roughly equal to the sum of the volumes of the gold particles initially grown on the respective nanocrystals (on average), before the coagulation was induced by iodine. However, we cannot exclude here that Ostwald ripening effects play also a role in dictating the overall size and domain structure of the gold nanoparticles in the assemblies.

In summary, our work introduces a general approach for the assembly of cadmium chalcogenide semiconductor nanocrystals by means of metallic gold junctions. It yields solely inorganic nanocrystalline assemblies, which are stable in organic solvents and hence should be easily processable for the fabrication of new nanocomposites and devices. Charge carrier transport should be facilitated across such a superstructure of Au-interlinked nanocrystals, and in addition novel structures with interesting mechanical properties and/or controlled porosity could be realized, which, in the case of tetrapod assemblies, could also serve as "scaffolds".

\section{Experimental}

Synthesis: All types of shape-controlled nanocrystals were synthesized following a previously described seeded-growth method [3c]. The preparation of Au-tipped nanocrystals involved the reduction of $\mathrm{AuCl}_{3}$ on the tips of the nanocrystals mediated by dodecylamine, in the presence of tetraoctylammonium bromide as stabilizer. We followed the procedure published by Banin et al. with slight modifications [6a]. A few $\mu \mathrm{L}$ of a diluted solution of $I_{2}$ in toluene $\left(0.1 \%\right.$ of $I_{2}$ in weight) were added to diluted solutions $(500 \mu \mathrm{L})$ of Au-tipped bullet-shaped nanocrystals (ca. $3 \mu \mathrm{M})$, or CdSe nanorods (ca. $0.1 \mu \mathrm{M}$ ), or tetrapods (ca. $0.1 \mu \mathrm{M}$ ), with a final $\mathrm{I}_{2}$ :Autipped nanocrystal molar ratio of ca. 15, and left to react at room temperature for approximately $30 \mathrm{~min}$ (see Experimental Section of the SI for more details). The exact volume of iodine solution added to the nanocrystal solution allowed us to tune the degree of assembly of the nanocrystals. At the end of the reaction, the assembled structures were purified by adding small amounts of methanol followed by centrifugation. The precipitate was soluble in nonpolar solvents such as toluene, hexane, or chloroform.

Instrumentation: Low-magnification TEM images were recorded with a JEOL JEM 1011 microscope operating at an accelerating voltage of $100 \mathrm{kV}$. Phase-contrast HRTEM measurements were performed with a JEOL $2100 \mathrm{~F}$ microscope, equipped with a field emission gun and working at an accelerating voltage of $200 \mathrm{kV}$. Annular dark field images for tomography reconstruction were collected in scanning mode (STEM) on a JEOL $3000 \mathrm{~F}$ field emission microscope with an accelerating voltage of $300 \mathrm{kV}$, by tilting the sample from $-65^{\circ}$ to $+65^{\circ}$ at $2^{\circ}$ steps. The samples for analysis were prepared by dropping a diluted solution of the sample on carbon-coated copper grids and then allowing the solvent to evaporate. Absorption measurements were carried out using a CARY 500 UV-vis spectrophotometer and fluorescence measurements were carried out using a CARY Eclipse fluorimeter.

\section{Acknowledgements}

This work was supported by the European project SA-NANO (Contract nr STRP 013698) and ESTEEM (Contract nr. 026019), and by the Italian Ministry of Research (under contract nr. RBLA03ER38 and FAR-DM 18604). S.B. and G.B. are grateful to the Fund for Scientific Research-Flanders. We would like to thank Dr. S. Shankar for many inspiring discussions. A.F. and I.R.F. have contributed equally to this work. Supporting Information is available online from Wiley InterScience or from the author.

Received: July 8, 2008

Revised: August 19, 2008

Published online: November 14, 2008

[1] a) H. Zhang, E. W. Edwards, D. Wang, H. Möhwald, Phys. Chem. Chem Phys. 2006, 8, 3288. b) S. Kinge, M. Crego-Calama, D. N. Reinhoudt, Chem. Phys. Chem. 2008, 9, 20.

[2] a) S. Ahmed, K. M. Ryan, Nano Lett. 2007, 7, 2480. b) K. M. Ryan, A Mastroianni, K. A. Stancil, H. Liu, A. P. Alivisatos, Nano Lett. 2006, 6, 1479. 
[3] a) D. V. Talapin, E. V. Shevchenko, C. B. Murray, A. Kornowski, S. Förster, H. Weller, J. Am. Chem. Soc. 2004, 126, 12984. b) L.-S. Li, A. P. Alivisatos, Adv. Mater. 2003, 15, 408. c) L. Carbone, C. Nobile, M. De Giorgi, F. Della Sala, G. Morello, P. Pompa, M. Hytch, E. Snoeck, A. Fiore, I. R. Franchini, M. Nadasan, A. F. Silvestre, L. Chiodo, S. Kudera, R. Cingolani, R. Krahne, L. Manna, Nano Lett. 2007, 7, 2942. d) B. Sun, H. Sirringhaus, J. Am. Chem. Soc. 2006, 128, 16231.

[4] D. Chen, L. Gao, J. Cryst. Growth 2004, 264, 216.

[5] a) K. K. Caswell, J. N. Wilson, U. H. F. Bunz, C. J. Murphy, J. Am. Chem. Soc. 2003, 125, 13914. b) K. G. Thomas, S. Barazzouk, B. I. Ipe, S. T. S. Joseph, P. V. Kamat, J. Phys. Chem. B 2004, 108, 13066. c) B. Pan, L. Ao, F. Gao, H. Tian, R. He, D. Cui, Nanotechnol. 2005, 16, 1776. d) Z. Nie, D. Fava, E. Kumacheva, S. Zou, G. C. Walker, M. Rubinstein, Nat. Mater. 2007, 6, 609.

[6] a) T. Mokari, E. Rothenberg, I. Popov, R. Costi, U. Banin, Science 2004, 304, 1787. b) A. Salant, E. Amitay-Sadovsky, U. Banin, J. Am. Chem. Soc. 2006, 128, 10006.

[7] a) M. C. Newton, P. A. Warburton, Mater. Today 2007, 10, 50. b) L. Manna, D. J. Milliron, A. Meisel, E. C. Scher, A. P. Alivisatos, Nat. Mater. 2003, 2, 382.
[8] L. Fang, J. Y. Park, Y. Cui, A. P. Alivisatos, J. Shcrier, B. Lee, L.-W. Wang, M. Salmeron, J. Chem. Phys. 2007, 127, 184704.

[9] Y. Cui, M. T. Björk, J. A. Liddle, C. Sönnichsen, B. Boussert, A. P. Alivisatos, Nano Lett. 2004, 4, 1093.

[10] W. Cheng, S. Dong, E. Wang, Angew. Chem. Int. Ed. 2003, 42, 449.

[11] A. Rai, A. Singh, A. Ahmad, M. Sastry, Langmuir 2006, 22, 736.

[12] S. Singh, R. Pasricha, U. M. Bhatta, P. V. Satyam, M. Sastry, B. L. V. Prasad, J. Mater. Chem. 2007, 17, 1614.

[13] A. Umeno, K. Hirakawa, Appl. Phys. Lett. 2005, 86, 143103.

[14] Y. Yasutake, K. Kono, M. Kanehara, T. Teranishi, M. R. Buitelaar, C. G. Smith, Y. Majima, Appl. Phys. Lett. 2007, 91, 203107.

[15] F. A. Cotton, G. Wilkinson, C. A. Murillo, M. Bochmann, Advanced Inorganic Chemistry, $6^{\text {th }}$ Ed., Wiley-Interscience, New York 1999, pp. 1098-1102.

[16] K. J. McGrew, J. W. Murphy, United States Patent 4557759, 1985.

[17] a) B. V. Enüstün, J. Turkevich, J. Am. Chem. Soc. 1963, 85, 3317. b) N. Satoh, H. Hasegawa, K. Tsujii, K. Kimura, J. Phys. Chem. 1994, 98, 2143. c) F. Spahn, N. Albers, M. Sremčević, C. Thornton, Europhys. Lett. 2004, 67, 545. 